\title{
On the growth of powers of operators with spectrum contained in Cantor sets
}

\author{
AGRAFEUIL Cyril
}

\begin{abstract}
For $\xi \in\left(0, \frac{1}{2}\right)$, we denote by $E_{\xi}$ the perfect symmetric set associated to $\xi$, that is

$$
E_{\xi}=\left\{\exp \left(2 i \pi(1-\xi) \sum_{n=1}^{+\infty} \epsilon_{n} \xi^{n-1}\right): \epsilon_{n}=0 \text { or } 1 \quad(n \geq 1)\right\}
$$
\end{abstract}

Let $s$ be a nonnegative real number, and $T$ be an invertible bounded operator on a Banach space with spectrum included in $E_{\xi}$. We show that if

$$
\begin{aligned}
& \left\|T^{n}\right\|=O\left(n^{s}\right), n \rightarrow+\infty \\
& \text { and } \quad\left\|T^{-n}\right\|=O\left(e^{n^{\beta}}\right), n \rightarrow+\infty \text { for some } \beta<\frac{\log \frac{1}{\xi}-\log 2}{2 \log \frac{1}{\xi}-\log 2} \text {, }
\end{aligned}
$$

then for every $\varepsilon>0, T$ satisfies the stronger property

$$
\left\|T^{-n}\right\|=O\left(n^{s+\frac{1}{2}+\varepsilon}\right), n \rightarrow+\infty .
$$

This result is a particular case of a more general result concerning operators with spectrum satisfying some geometrical conditions.

\section{Introduction}

We denote by $\mathbb{T}$ the unit circle and by $\mathbb{D}$ the open unit disk. We shall say that a closed subset $E$ of $\mathbb{T}$ is a $K$-set if there exists a positive constant $c$ such that for any arc $L$ of $\mathbb{T}$,

$$
\sup _{z \in L} d(z, E) \geq c|L|
$$

where $|L|$ denotes the length of the $\operatorname{arc} L$ and $d(z, E)$ the distance between $z$ and $E$. Let $E$ be a $K$-set. We set

$$
\delta(E)=\sup \left\{\delta \geq 0: \int_{0}^{2 \pi} \frac{1}{d\left(e^{i t}, E\right)^{\delta}} \mathrm{d} t<+\infty\right\} .
$$

We have $\delta(E) \geq \frac{\log \frac{1}{1-c}}{\log \frac{2}{1-c}}$ (see [2] section 5, proof of lemma 2 and corollary). E. M. Dyn'kin showed in 2] that condition $(K)$ characterizes the interpolating sets for $\Lambda_{s}^{+}(\mathbb{T}), s>0$ (see 
section 2 for the definition of $\left.\Lambda_{s}^{+}(\mathbb{T})\right)$. Let $s$ be a nonnegative real number, and let $T$ be an invertible operator on a Banach space. We show (theorem 2.3) that if the spectrum of $T$ is included in $E$ and if $T$ satisfies

$$
\begin{aligned}
& \left\|T^{n}\right\|=O\left(n^{s}\right), n \rightarrow+\infty \\
& \text { and }\left\|T^{-n}\right\|=O\left(e^{n^{\beta}}\right), n \rightarrow+\infty \text { for some } \beta<\frac{\delta(E)}{1+\delta(E)} \text {, }
\end{aligned}
$$

then for every $\varepsilon>0, T$ also satisfies the stronger property

$$
\left\|T^{-n}\right\|=O\left(n^{s+\frac{1}{2}+\varepsilon}\right), n \rightarrow+\infty .
$$

For $\xi \in\left(0, \frac{1}{2}\right)$, we denote by $E_{\xi}$ the perfect symmetric set associated to $\xi$, that is

$$
E_{\xi}=\left\{\exp \left(2 i \pi(1-\xi) \sum_{n=1}^{+\infty} \epsilon_{n} \xi^{n-1}\right): \epsilon_{n}=0 \text { or } 1 \quad(n \geq 1)\right\}
$$

We set $b(\xi)=\frac{\log \frac{1}{\xi}-\log 2}{2 \log \frac{1}{\xi}-\log 2}$. We obtain (as a consequence of theorem 2.3) that if the spectrum of $T$ is included in $E_{\xi},\left\|T^{n}\right\|=O\left(n^{s}\right), n \rightarrow+\infty$ and $\left\|T^{-n}\right\|=O\left(e^{n^{\beta}}\right), n \rightarrow+\infty$ for some $\beta<b(\xi)$, then $T$ satisfies (11). Notice that J. Esterle showed in [5] that if $T$ is a contraction on a Banach space (respectively on a Hilbert space) with spectrum included in $E_{\frac{1}{q}}\left(\right.$ respectively included in $\left.E_{\xi}\right)$ such that $\left\|T^{-n}\right\|=O\left(e^{n^{\beta}}\right), n \rightarrow+\infty$ for some $\beta<b\left(\frac{1}{q}\right)$ (respectively $\beta<b(\xi)$ ), then $\sup _{n \geq 0}\left\|T^{-n}\right\|<+\infty$ (respectively $T$ is an isometry). Here $q$ is an integer greater than or equal to 3.

\section{Growth of powers of operators}

Let $p$ be a non-negative integer. We denote by $\mathcal{C}^{p}(\mathbb{T})$ the space of $p$ times continuously differentiable functions on $\mathbb{T}$. We set

$$
a^{p}(\mathbb{D})=\left\{f \in \mathcal{C}^{p}(\mathbb{T}): \widehat{f}(n)=0 \quad(n<0)\right\},
$$

$\mathcal{C}^{\infty}(\mathbb{T})=\bigcap_{p \geq 0} \mathcal{C}^{p}(\mathbb{T})$ and $a^{\infty}(\mathbb{D})=\bigcap_{p \geq 0} a^{p}(\mathbb{D})$

Let $s$ be a nonnegative real number, we denote by $[s]$ the nonnegative integer such that $[s] \leq s<[s]+1$. We define the Banach algebra

$$
\Lambda_{s}(\mathbb{T})=\left\{f \in \mathcal{C}^{[s]}(\mathbb{T}): \sup _{z, z^{\prime} \in \mathbb{T}} \frac{\left|f^{([s])}(z)-f^{([s])}\left(z^{\prime}\right)\right|}{\left|z-z^{\prime}\right|^{s-[s]}}<+\infty\right\},
$$


equiped with the norm $\|f\|_{\Lambda_{s}}=\|f\|_{\mathcal{C}^{[s]}(\mathbb{T})}+\sup _{z, z^{\prime} \in \mathbb{T}} \frac{\left|f^{([s])}(z)-f^{([s])}\left(z^{\prime}\right)\right|}{\left|z-z^{\prime}\right|^{s-[s]}}$. We also define the subalgebra

$$
\lambda_{s}(\mathbb{T})=\left\{f \in \mathcal{C}^{[s]}(\mathbb{T}):\left|f^{([s])}(z)-f^{([s])}\left(z^{\prime}\right)\right|=o\left(\left|z-z^{\prime}\right|^{s-[s]}\right),\left|z-z^{\prime}\right| \rightarrow 0\right\},
$$

which we equip with the same norm. We also set

$$
\begin{aligned}
\Lambda_{s}^{+}(\mathbb{T}) & =\left\{f \in \Lambda_{s}(\mathbb{T}): \widehat{f}(n)=0 \quad(n<0)\right\} \\
\text { and } \lambda_{s}^{+}(\mathbb{T}) & =\left\{f \in \lambda_{s}(\mathbb{T}): \widehat{f}(n)=0 \quad(n<0)\right\} .
\end{aligned}
$$

We remark that if $s$ is an integer, $\Lambda_{s}(\mathbb{T})=\lambda_{s}(\mathbb{T})=\mathcal{C}^{s}(\mathbb{T})$ and so $\Lambda_{s}^{+}(\mathbb{T})=\lambda_{s}^{+}(\mathbb{T})=a^{s}(\mathbb{D})$. We define

$$
N_{s}(E)=\left\{f \in \Lambda_{s}(\mathbb{T}): f_{\left.\right|_{E}}=\ldots=f_{\left.\right|_{E}}^{([s])}=0\right\},
$$

and set $N_{s}^{+}(E)=N_{s}(E) \cap \Lambda_{s}^{+}(\mathbb{T})$.

Lemma 2.1. Let $s$ be a nonnegative real number. Then for all $\varepsilon>0$, we have the following continuous embedding

$$
\Lambda_{s+\frac{1}{2}+\varepsilon}(\mathbb{T}) \hookrightarrow A_{s}(\mathbb{T})
$$

Proof. For $s=0$, this is a result of Bernstein (see 9], p.13). The general case is obtained by the same arguments. Let $\varepsilon>0$, and set $\tilde{s}=s+\frac{1}{2}+\varepsilon$. Let $f \in \Lambda_{\tilde{s}}(\mathbb{T})$. For $h>0$, define

$$
P(h)=\int_{0}^{2 \pi}\left|f^{([\tilde{s}])}\left(e^{i(t-h)}\right)-f^{([\tilde{s}])}\left(e^{i(t+h)}\right)\right|^{2} \mathrm{~d} t .
$$

It follows from Parseval equality that

$$
P(h)=8 \pi \sum_{n=-\infty}^{+\infty}|\widehat{f([\tilde{s}])}(n)|^{2} \sin ^{2}(n h) .
$$

Let $j_{0}$ be the smallest integer such that $[\tilde{s}]<2^{j_{0}}$ and let $j \geq j_{0}$. It follows from the relation $\widehat{f^{([\tilde{s}])}}(n)=\left(\prod_{k=1}^{[\tilde{s}]}(n+k)\right) \widehat{f}(n+[\tilde{s}])(n \in \mathbb{Z})$ and from (2) that there exists a constant $C_{1}>0$ independent of $f$ such that

$$
P(h) \geq \frac{4}{C_{1}^{2}} \sum_{|n|=2^{j}}^{2^{j+1}-1}|\widehat{f}(n+[\tilde{s}])|^{2}(1+|n|)^{2[\tilde{s}]} \sin ^{2}(n h) .
$$

Using the Cauchy-Schwartz inequality, we have

$$
\sum_{|n|=2^{j}}^{2^{j+1}-1}|\widehat{f}(n+[\tilde{s}])|(1+|n|)^{s} \leq\left(\sum_{|n|=2^{j}}^{2^{j+1}-1}|\widehat{f}(n+[\tilde{s}])|^{2}(1+|n|)^{2[\tilde{s}]}\right)^{\frac{1}{2}}\left(\sum_{n=2^{j}}^{2^{j+1}-1}(1+|n|)^{2 s-2[\tilde{s}]}\right)^{\frac{1}{2}}
$$


Set $h=\frac{\pi}{3.2^{j}}$. For all integers $n$ such that $2^{j} \leq|n| \leq 2^{j+1}-1$, we have $\frac{\pi}{3} \leq|n h| \leq \frac{2 \pi}{3}$, and so $\sin ^{2}(n h) \geq \frac{1}{4}$. So, we deduce from (3) that

$$
\left(\sum_{|n|=2^{j}}^{2^{j+1}-1}|\widehat{f}(n+[\tilde{s}])|^{2}(1+|n|)^{2[\tilde{s}]}\right)^{\frac{1}{2}} \leq C_{1} P\left(\frac{\pi}{3.2^{j}}\right)^{\frac{1}{2}}
$$

Then, as $f \in \Lambda_{\tilde{s}}(\mathbb{T})$, we have

$$
P\left(\frac{\pi}{3.2^{j}}\right)^{\frac{1}{2}} \leq(2 \pi)^{\frac{1}{2}}\|f\|_{\Lambda_{\tilde{s}}}\left(\frac{2 \pi}{3.2^{j}}\right)^{\tilde{s}-[\tilde{s}]}
$$

so that

$$
\left(\sum_{|n|=2^{j}}^{2^{j+1}-1}|\widehat{f}(n+[\tilde{s}])|^{2}(1+|n|)^{2[\tilde{s}]}\right)^{\frac{1}{2}} \leq C_{1}(2 \pi)^{\frac{1}{2}}\|f\|_{\Lambda_{\tilde{s}}}\left(\frac{2 \pi}{3.2^{j}}\right)^{\tilde{s}-[\tilde{s}]}
$$

Furthermore, there exists a constant $C_{2}>0$ such that

$$
\left(\sum_{|n|=2^{j}}^{2^{j+1}-1}(1+|n|)^{2 s-2[\tilde{s}]}\right)^{\frac{1}{2}} \leq C_{2} 2^{j\left(s-[\tilde{s}]+\frac{1}{2}\right)} .
$$

Finally we deduce from (44) and the inequalities (5) and (6) that there exists a constant $C_{3}>0$ independent of $f$ such that for all $j \geq j_{0}$,

$$
\sum_{|n|=2^{j}}^{2^{j+1}-1}|\widehat{f}(n+[\tilde{s}])|(1+|n|)^{s} \leq 2^{j\left(s-\tilde{s}+\frac{1}{2}\right)} C_{3}\|f\|_{\Lambda_{\tilde{s}}}=2^{-\varepsilon j} C_{3}\|f\|_{\Lambda_{\tilde{s}}} .
$$

Summing over $j \geq j_{0}$ these inequalities, we get

$$
\sum_{|n| \geq 2^{j_{0}}}|\widehat{f}(n+[\tilde{s}])|(1+|n|)^{s} \leq \frac{C_{3}}{1-2^{-\varepsilon}}\|f\|_{\Lambda_{\tilde{s}}}
$$

On the other hand, we have $|\widehat{f}(n)| \leq\|f\|_{\Lambda_{\tilde{s}}}$ for every $n \in \mathbb{Z}$. So, since $j_{0}$ is independent of $f$, there exists a constant $K>0$ (independent of $f$ ) such that

$$
\|f\|_{s} \leq K\|f\|_{\Lambda_{\tilde{s}}}
$$

Before giving the main theorem of the paper, we need the following lemma. 
Lemma 2.2. Let $E$ be a closed subset of $\mathbb{T}$. We assume that there exists $\delta>0$ for which $\int_{0}^{2 \pi} \frac{1}{d\left(e^{i t}, E\right)^{\delta}} \mathrm{d} t<+\infty$. Let $\beta<\frac{\delta}{1+\delta}$ and let $T$ be an invertible operator on a Banach space with spectrum included in $E$ that satisfies

$$
\begin{aligned}
& \left\|T^{n}\right\|=O\left(n^{s}\right), n \rightarrow+\infty \quad(\text { for some nonnegative real } s) \\
& \text { and }\left\|T^{-n}\right\|=O\left(e^{n^{\beta}}\right), n \rightarrow+\infty,
\end{aligned}
$$

Then there exists an outer function $f \in a^{\infty}(\mathbb{D})$ which vanishes exactly on $E$ and such that $f(T):=\sum_{n=0}^{+\infty} \widehat{f}(n) T^{n}=0$.

Proof. Let $\omega$ be the weight defined by $\omega(n)=\left\|T^{n}\right\|(n \in \mathbb{Z})$. Let $\Phi$ be the continuous morphism from $A_{\omega}(\mathbb{T})$ to $\mathcal{L}(X)$ defined by

$$
\Phi(f)=f(T)=\sum_{n=-\infty}^{+\infty} \widehat{f}(n) T^{n} \quad\left(f \in A_{\omega}(\mathbb{T})\right) .
$$

Since the algebra $A_{\omega}(\mathbb{T})$ is regular, we have $\{z \in \mathbb{T}: f(z)=0 \quad(f \in \operatorname{Ker} \Phi)\} \subset E$ (see [7, theorem 2.5), and so $J_{\omega}(E) \subset \operatorname{Ker} \Phi$. Then the result follows from lemmas 7.1 and 7.2 of [5].

Theorem 2.3. Let $E$ be a $K$-set, and let s be a nonnegative real number. Then, any invertible operator $T$ on a Banach space with spectrum included in $E$ that satisfies

$$
\begin{aligned}
\left\|T^{n}\right\| & =O\left(n^{s}\right), n \rightarrow+\infty \\
\text { and }\left\|T^{-n}\right\| & =O\left(e^{n^{\beta}}\right), n \rightarrow+\infty \text { for some } \beta<\frac{\delta(E)}{1+\delta(E)},
\end{aligned}
$$

also satisfies the stronger property

$$
\left\|T^{-n}\right\|=O\left(n^{s+\frac{1}{2}+\varepsilon}\right), n \rightarrow+\infty,
$$

for all $\varepsilon>0$.

Proof. Let $\varepsilon>0$ and set $\tilde{s}=s+\frac{1}{2}+\varepsilon$. Without loss of generality, we may assume that $\tilde{s}$ is not an integer. Let $t$ a real number, which is not an integer, and satisfies $s+\frac{1}{2}<t<\tilde{s}$ and $[t]=[\tilde{s}]$. According to lemma 2.1] we can define a continuous morphism $\Phi$ from $\lambda_{t}^{+}(\mathbb{T})$ to $\mathcal{L}(X)$ by

$$
\Phi(f)=f(T)=\sum_{n=0}^{+\infty} \widehat{f}(n) T^{n} \quad\left(f \in \lambda_{t}^{+}(\mathbb{T})\right) .
$$

Let $I=\operatorname{Ker} \Phi, I$ is a closed ideal of $\lambda_{t}^{+}(\mathbb{T})$. We denote by $S_{I}$ its inner factor, that is the greatest common divisor of all inner factors of the non-zero functions in $I$ (see [8] p.85), and 
we set, for $0 \leq k \leq[t], h^{k}(I)=\left\{z \in \mathbb{T}: f(z)=\ldots=f^{(k)}(z)=0 \quad(f \in I)\right\}$.

F. A. Shamoyan showed in 12 that

$$
I=\left\{f \in \lambda_{t}^{+}(\mathbb{T}): S(I) \mid S(f) \text { and } f^{(k)}=0 \text { on } h^{k}(I) \text { for all } 0 \leq k \leq[t]\right\},
$$

where $S(f)$ denotes the inner factor of $f$ and $S(I) \mid S(f)$ means that $S(f) / S(I)$ is a bounded holomorphic function in $\mathbb{D}$. Since $\beta<\frac{\delta(E)}{1+\delta(E)}$, there exists $0<\delta<\delta(E)$ such that $\beta<\frac{\delta}{1+\delta}$. We have, by definition of $\delta(E), \int_{0}^{2 \pi} \frac{1}{d\left(e^{i t}, E\right)^{\delta}} \mathrm{d} t<+\infty$. So we deduce from lemma 2.2 that there exists an outer function $f \in a^{\infty}(\mathbb{D})$ which vanishes exactly on $E$ and such that $f \in I$. Therefore, we have $S(I)=1$ and $h^{0}(I) \subset E$, so that $N_{t}^{+}(E) \cap \lambda_{t}(\mathbb{T}) \subset I$. Now, as $\Lambda_{\tilde{s}}^{+}(\mathbb{T}) \subset \lambda_{t}^{+}(\mathbb{T})$, we can define a continuous morphism $\Psi$ from $\Lambda_{\tilde{s}}^{+}(\mathbb{T})$ to $\mathcal{L}(X)$ by $\Psi=\Phi_{\left.\right|_{\Lambda_{\tilde{s}}^{+}(\mathbb{T})}}$. Using what precedes, we have

$$
N_{\tilde{s}}^{+}(E) \subset \operatorname{Ker} \Psi \text {. }
$$

So there exists a continuous morphism $\tilde{\Psi}$ from $\Lambda_{\tilde{s}}^{+}(\mathbb{T}) / N_{\tilde{s}}^{+}(E)$ into $\mathcal{L}(X)$ such that $\Psi=\tilde{\Psi} \circ \pi_{\tilde{s}}^{+}$, where $\pi_{\tilde{s}}^{+}$is the canonical surjection from $\Lambda_{\tilde{s}}^{+}(\mathbb{T})$ to $\Lambda_{\tilde{s}}^{+}(\mathbb{T}) / N_{\tilde{s}}^{+}(E)$. Since $E$ is a $K$-set, by a theorem of E. M. Dyn'kin [2], it is an interpolating set for $\Lambda_{\tilde{s}}^{+}(\mathbb{T})$, so that the canonical imbedding $i$ from $\Lambda_{\tilde{s}}^{+}(\mathbb{T}) / N_{\tilde{s}}^{+}(E)$ into $\Lambda_{\tilde{s}}(\mathbb{T}) / N_{\tilde{s}}(E)$ is onto. We have, for $n \geq 0$,

$$
T^{-n}=\tilde{\Psi} \circ i^{-1} \circ \pi_{\tilde{s}}\left(\alpha^{-n}\right),
$$

where $\pi_{\tilde{s}}$ denote the canonical surjection from $\Lambda_{\tilde{s}}(\mathbb{T})$ to $\Lambda_{\tilde{s}}(\mathbb{T}) / N_{\tilde{s}}(E)$ and where $\alpha: z \rightarrow z$ is the identity map. So we have, for $n \geq 0$,

$$
\begin{aligned}
\left\|T^{-n}\right\| & \leq\left\|\tilde{\Psi} \circ i^{-1}\right\|\left\|\pi_{\tilde{s}}\left(\alpha^{-n}\right)\right\|_{\Lambda_{\tilde{s}}} \\
& \leq\left\|\tilde{\Psi} \circ i^{-1}\right\|(1+n)^{\tilde{s}},
\end{aligned}
$$

which completes the proof.

We give two immediate corollaries of this theorem.

Corollary 2.4. Let $\xi \in\left(0, \frac{1}{2}\right)$ and let $s$ be a nonnegative real number. Then, any invertible operator $T$ on a Banach space with spectrum included in $E_{\xi}$ that satisfies

$$
\begin{aligned}
& \left\|T^{n}\right\|=O\left(n^{s}\right), n \rightarrow+\infty \\
& \text { and }\left\|T^{-n}\right\|=O\left(e^{n^{\beta}}\right), n \rightarrow+\infty \text { for some } \beta<b(\xi),
\end{aligned}
$$

also satisfies the stronger property

$$
\left\|T^{-n}\right\|=O\left(n^{s+\frac{1}{2}+\varepsilon}\right), n \rightarrow+\infty
$$

for all $\varepsilon>0$. 
Proof. It is well known that $E_{\xi}$ is a $K$-set (see proposition 2.5 of [5]). Moreover, $E_{\xi}$ satisfies $\int_{0}^{2 \pi} \frac{1}{d\left(e^{i t}, E\right)^{\delta}} \mathrm{d} t<+\infty$ if and only if $\delta<1+\frac{\log 2}{\log \xi}$. Indeed, the condition $\int_{0}^{2 \pi} \frac{1}{d\left(e^{i t}, E\right)^{\delta}} \mathrm{d} t<$ $+\infty$ is equivalent to $\sum_{n=1}^{+\infty} \sum_{i=1}^{2^{n-1}}\left|L_{n, i}\right|^{1-\delta}$, where $L_{n, i}$ are the arcs contiguous to $E_{\xi}$, and $\left|L_{n, i}\right|$ are their length, which is equal to $2 \pi \xi^{n-1}(1-2 \xi)$ (see 10 for further details). Then it is easily seen that the last series converges if and only if $\delta<1+\frac{\log 2}{\log \xi}$, so $\delta\left(E_{\xi}\right)=1+\frac{\log 2}{\log \xi}$. Now, the result follows immediately from theorem 2.3

Then we obtain an other immediate result, which generalizes theorem 4.1 of [3]. Indeed, the condition " $\left\|T^{-n}\right\|=O\left(e^{n^{\beta}}\right), n \rightarrow+\infty$ " which appears in the following corollary is weaker than the condition used by the authors of $[3]$.

Corollary 2.5. Let $E$ be a $K$-set, and let $s$ be a nonnegative real number. Then, there exists a constant $\beta>0$ independent of $s$ such that any invertible operator $T$ on a Banach space with spectrum included in $E$ that satisfies

$$
\begin{gathered}
\left\|T^{n}\right\|=O\left(n^{s}\right), n \rightarrow+\infty \\
\text { and }\left\|T^{-n}\right\|=O\left(e^{n^{\beta}}\right), n \rightarrow+\infty,
\end{gathered}
$$

also satisfies the stronger property

$$
\left\|T^{-n}\right\|=O\left(n^{s+\frac{1}{2}+\varepsilon}\right), n \rightarrow+\infty
$$

for all $\varepsilon>0$.

Proof. As $E$ is a $K$-set, we deduce from [2] (section 5, corollary) that $\delta(E)>0$. Then the result follows immediately from theorem 2.3 with any $\beta<\frac{\delta(E)}{1+\delta(E)}$.

\section{Remark 2.6:}

1) Some results concerning operators with countable spectrum are obtained in [13] and in [1]. Let $E$ be a closed subset of $\mathbb{T}$ and let $s, t$ be two nonnegative reals. We denote by $P(s, t, E)$ the following property: every invertible operator $T$ on a Banach space such that $\operatorname{Sp} T \subset E$ and satisfies the conditions:

$$
\begin{aligned}
& \left\|T^{n}\right\|=O\left(n^{s}\right)(n \rightarrow+\infty) \\
& \left\|T^{-n}\right\|=O\left(e^{\varepsilon \sqrt{n}}\right)(n \rightarrow+\infty), \text { for all } \varepsilon>0,
\end{aligned}
$$

also satisfies the stronger property

$$
\left\|T^{-n}\right\|=O\left(n^{t}\right)(n \rightarrow+\infty) .
$$

M. Zarrabi showed in [13] (théorème 3.1 and remarque 2.a) that a closed subset $E$ of $\mathbb{T}$ satisfies $P(0,0, E)$ if and only if $E$ is countable. Notice that $E$ is called a Carleson set if $\int_{0}^{2 \pi} \log ^{+} \frac{1}{d\left(e^{i t}, E\right)} \mathrm{d} t<+\infty$. If $E$ is a countable closed subset of $\mathbb{T}$, we show in [1] that the 
following conditions are equivalent:

(i) there exist two positive constants $C_{1}, C_{2}$ such that for every $\operatorname{arc} I \subset \mathbb{T}$,

$$
\frac{1}{|I|} \int_{I} \log ^{+} \frac{1}{d\left(e^{i t}, E\right)} \mathrm{d} t \leq C_{1} \log \frac{1}{|I|}+C_{2}
$$

(ii) $E$ is a Carleson set and for all $s \geq 0$, there exists $t$ such that $P(s, t, E)$ is satisfied. For contractions with spectrum satisfying the Carleson condition, we can see [11].

2) When $\xi=\frac{1}{q}$, the constant $b\left(\frac{1}{q}\right)$ in corollary [2.4 is the best possible in view of [6], where the authors built a contraction $T$ such that $\lim _{n \rightarrow+\infty} \log \left\|T^{-n}\right\|=+\infty, S p T \subset E_{\frac{1}{q}}$ and $\log \left\|T^{-n}\right\|=O\left(n^{b\left(\frac{1}{q}\right)}\right)$. According to theorem 6.4 of [4], $T$ doesn't satisfy $\left\|T^{-n}\right\|=O\left(n^{s}\right)$ for any real $s \geq 0$.

\section{References}

[1] C. Agrafeuil, Idéaux fermés de certaines algèbres de Beurling et application aux opérateurs à spectre dénombrable, preprint.

[2] E. M. Dynkin, Free interpolation set for Hölder classes, Mat. Sbornik, 109 (1979), 107128.

[3] O. El-Fallah et K. Kellay, Sous-espaces biinvariants pour certains shifts pondérés, Ann. Inst. Fourier 48 (1998), no. 5, 1543-1558.

[4] J. Esterle, Uniqueness, strong form of uniqueness and negative powers of contractions, Banach Center Publ. 30 (1994), 1-19.

[5] J. Esterle, Distributions on Kronecker sets, strong forms of uniqueness, and closed ideals of $A^{+}$, J. reine angew. Math. 450 (1994), 43-82.

[6] J. Esterle, M. Rajoelina and M. Zarrabi, On contractions with spectrum contained in Cantor set, Math. Proc. Camb. Phil. Soc. 117 (1995), 339-343.

[7] J. Esterle, E. Strouse and F. Zouakia, Theorems of Katznelson-Tzafriri type for contractions, J. Func. Anal. (2) 94 (1990), 273-287.

[8] K. Hoffman "Banach spaces of analytic functions", Prentic-Hall, Englewood Cliffs, 1962.

[9] J. P. Kahane, "Séries de Fourier absolument convergentes", Erg. Math. 336, Springer Verlag, Berlin-Heidelberg-New York, 1973.

[10] J. P. Kahane, R. Salem, "Ensembles parfaits et séries trigonométriques", Paris, Hermann, 1963.

[11] K. Kellay, Contractions et hyperdistributions à spectre de Carleson, J. London Math. Soc. (2) 58 (1998), 185-196. 
[12] F. A. Shamoyan Closed ideals in algebras of functions analytic in the disc and smooth up to its boundary, Mat. Sbornik 79 (1994), 425-445.

[13] M. Zarrabi, Contractions à spectre dénombrable et propriété d'unicité des fermés dénombrables du cercle unité, Ann. Inst. Fourier 43 (1993), 251-263.

2000 Mathematics Subject Classification: 46J15, 46J20, 47A30.

Key-words: operators, Beurling algebra, spectral synthesis, perfect symmetric set.

\section{AGRAFEUIL Cyril}

Cyril.Agrafeuil@math.u-bordeaux.fr

Laboratoire Bordelais d'Analyse et Géométrie (LaBAG), CNRS-UMR 5467 Université Bordeaux I

351 , cours de la libération

33405 Talence cedex, FRANCE. 